\title{
Bronquite plástica em criança com talassemia alfa
}

\author{
Plastic bronchitis in a child with thalassemia alpha
}

\author{
Tiago N. $\operatorname{Veras}^{1}$, Gustavo M. Lannes ${ }^{1}$, Jefferson P. Piva ${ }^{2}$, Renato T. Stein ${ }^{3}$, \\ Marcus H. Jones ${ }^{3}$, Vinicius D. Silva4 ${ }^{4}$ Pedro Celiny R. Garcia ${ }^{5}$, Paulo M. C. Pitrez ${ }^{3}$
}

\section{Resumo}

Objetivo: A bronquite plástica é uma doença infreqüente na criança, sendo caracterizada por moldes ou cilindros mucofibrinosos na árvore traqueobrônquica. Faz parte do diagnóstico diferencial de crianças com insuficiência respiratória de início agudo, e o tratamento precoce é importante para a resolução do quadro. O objetivo deste relato é descrever um caso de bronquite plástica tratado com sucesso por endoscopia, em paciente portador de talassemia alfa.

Descrição: Criança do sexo masculino, 3 anos de idade, sem antecedentes mórbidos significativos, apresentou quadro de insuficiência respiratória aguda, com achados radiológicos de atelectasia pulmonar sugestivos de aspiração de corpo estranho. Não havia sintomas respiratórios ou antecedentes de alergia ou infecções respiratórias de repetição. A realização de broncoscopia flexível, complementada por endoscopia rígida e exame anatomopatológico, evidenciou a presença de bronquite plástica. Após a realização da endoscopia, a criança evoluiu satisfatoriamente, com curva térmica afebril e extubação em 72 horas. Foram utilizadas medicações sintomáticas, sem necessidade de antimicrobianos. Dez dias após a alta, a radiografia de tórax encontrava-se normal. A talassemia alfa foi diagnosticada através da eletroforese de hemoglobina.

Comentários: A importância clínica da bronquite plástica reside no fato de apresentar um quadro semelhante ao de outras doenças mais prevalentes, como a aspiração de corpo estranho e a asma brônquica. A suspeita do quadro recomenda a realização de endoscopia para diagnóstico e tratamento. É reconhecida a ocorrência de bronquite plástica em crianças com fibrose cística, pós-operatório de cirurgia cardíaca e anemia falciforme. No presente artigo, foi observada uma associação com talassemia alfa.

J Pediatr (Rio J). 2005;81(6):499-502: Bronquite plástica, corpo estranho, broncoscopia, talassemia.

1. Médico residente de Pneumologia Pediátrica, Hospital São Lucas, Pontifícia Universidade Católica do Rio Grande do Sul (PUCRS), Porto Alegre, RS.

2. Professor adjunto, Departamento de Pediatria, Faculdade de Medicina, PUCRS, Porto Alegre, RS. Professor adjunto, Universidade Federal do Rio Grande do Sul (UFRGS), Porto Alegre, RS.

3. Professor adjunto, Departamento de Pediatria, Faculdade de Medicina, PUCRS, Porto Alegre, RS.

4. Professor adjunto, Departamento de Patologia e Radiações, Faculdade de Medicina, PUCRS, Porto Alegre, RS.

5. Professor adjunto, Departamento de Pediatria, PUCRS, Porto Alegre, RS. Chefe da UTI pediátrica, Hospital São Lucas, PUCRS.

Artigo submetido em 28.06.05, aceito em 28.09.05.

Como citar este artigo: Veras TN, Lannes GM, Piva JP, Stein RT, Jones $\mathrm{MH}$, Silva VD, et al. Bronquite plástica em criança com talassemia alfa. J Pediatr (Rio J). 2005;81:499-502.

\section{Abstract}

Objective: Plastic bronchitis is an unusual condition in children, associated with formation of mucofibrinous casts and mucous plugging of the tracheobronchial tree. Given that this illness is part of the differential diagnosis of acute respiratory failure, early treatment is important for improved prognosis. The aim of this report is to describe a case of plastic bronchitis in a child with alpha-thalassemia that was treated successfully with endoscopy.

Description: A 3 year old, black, male child, previously healthy, presented with acute respiratory failure and a chest $\mathrm{x}$-ray showing pulmonary atelectasis. There was no evidence of respiratory symptoms or previous allergy state. The diagnosis of plastic bronchitis was made using flexible and rigid bronchoscopy, and confirmed by histopathologic findings. The child progressed well, treatment was based on supportive care and antibiotics were not used. Ten days after discharge, radiographic appearance was normal. Alpha thalassemia was diagnosed through hemoglobin electrophoresis.

Comments: Plastic bronchitis is clinically important because has similar presentation to other prevalent diseases, such as foreign body aspiration and asthma. When plastic bronchitis is suspected, endoscopy is indicated in order to confirm diagnosis and define treatment. Plastic bronchitis has been previously described in patients with cystic fibrosis, cardiac surgery and sickle cell disease. In this case, an association with alpha-thalassemia was observed.

J Pediatr (Rio J). 2005;81(6):499-502: Plastic bronchitis, foreign body, bronchoscopy, thalassemia.

\section{Introdução}

Bronquite plástica (BP) é uma doença infreqüente, em que ocorre a impacção ou moldagem da árvore traqueobrônquica por cilindros mucofibrinosos, raramente constituindo doença primária ${ }^{1}$. A BP está mais associada, na faixa etária pediátrica, com doenças pulmonares inflamatórias ou com aumento de secreção, como a asma, fibrose cística e infecções pulmonares ${ }^{2}$. Relatos recentes mostram, também, uma associação com pacientes com anemia falciforme (síndrome torácica aguda), cardiopatias congênitas e submetidos a procedimentos cirúrgicos cardiológicos 2,3 .

Apesar de a BP ser uma condição infreqüente, sua importância maior reside no fato de poder apresentar um 
quadro clínico muito semelhante ao de doenças de elevada prevalência, porém com necessidade de um diagnóstico precoce e específico para obter a resolução clínica.

No presente relato, os autores descrevem um caso de paciente pediátrico admitido por insuficiência respiratória aguda, de instalação súbita, com suspeita de aspiração de corpo estranho, o qual resultou em diagnóstico de BP associada à talassemia alfa.

\section{Descrição do caso}

Paciente de 3 anos de idade, preto, previamente hígido, foi admitido no serviço de urgência do Hospital São Lucas da PUCRS, no dia 18 de janeiro de 2005, com quadro de dificuldade respiratória e febre há 24 horas. Não havia relato de pródromos virais ou história prévia de alergias ou infecções respiratórias de repetição. Ao exame físico, apresentava taquipnéia (freqüência respiratória de 50 ipm), retrações subcostais e intercostais, tempo expiratório aumentado e murmúrio vesicular abolido em hemitórax esquerdo. Havia hipoxemia, a saturação de oxigênio estava em $88-90 \%$ em ar ambiente.

A radiografia de tórax mostrava hiperinsuflação bilateral, atelectasia parcial do pulmão esquerdo, com desvio do mediastino para o lado ipsilateral e discreto infiltrado intersticial bilateral (Figura 1).

O hemograma da admissão demonstrava anemia microcítica (hematócrito: $27 \%$, hemoglobina: 9,1 g/dl, volume corpuscular médio: $64 \mu^{3}$ ), com leucócitos totais de 16.500 céls $/ \mathrm{mm}^{3}$ (bastonetes: $38,5 \%$, segmentados: $49,5 \%$ e linfócitos: $8 \%$ ). A gasometria arterial em ar ambiente apresentava $\mathrm{pH}: 7,37, \mathrm{pCO}_{2}: 21 \mathrm{mmHg}, \mathrm{pO}_{2}: 61 \mathrm{mmHg}$, $\mathrm{HCO} 312$ e $\mathrm{SaO}_{2} 99 \%$.

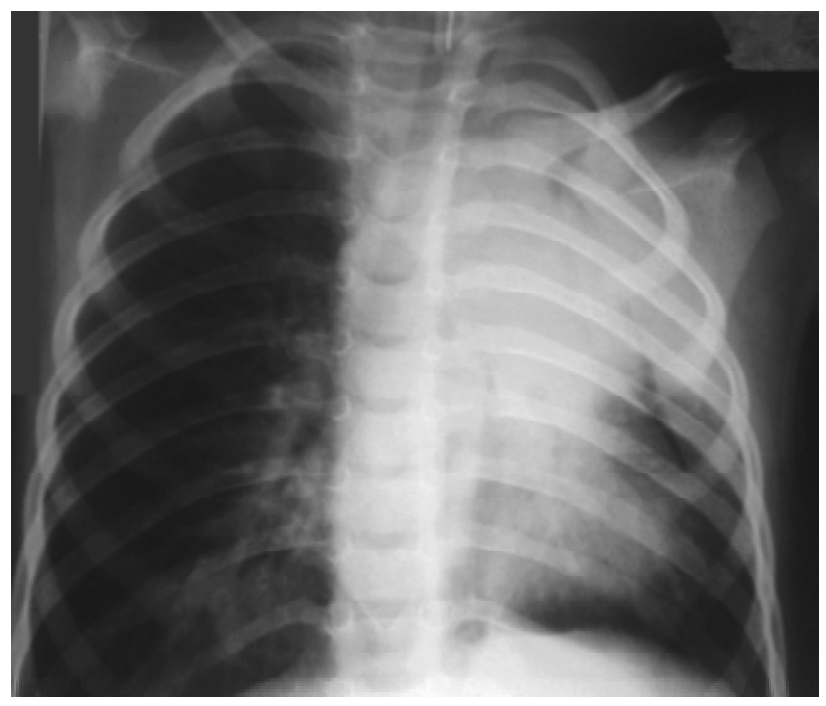

Figura 1 - Radiograma de tórax com hiperinsuflação bilateral, atelectasia parcial do pulmão esquerdo, com desvio do mediastino para o lado ipsilateral e discreto infiltrado intersticial bilateral
O paciente evoluiu para insuficiência respiratória, sendo colocado em ventilação mecânica em unidade de terapia intensiva. Os parâmetros de ventilação com o aparelho Sechrist IV eram PIP 35; PEEP 6; FR 20 ipm e $\mathrm{FiO}_{2}$ 40\%. Devido à suspeita de aspiração de corpo estranho, foi submetido a broncoscopia flexível de urgência na própria unidade de terapia intensiva, na qual se evidenciou obstrução total de brônquio principal esquerdo por secreção muito espessa e de aspecto "caseoso". Após múltiplas tentativas de aspiração brônquica, inclusive com uso de $\mathrm{N}$-acetilcisteína tópica diluída, houve remoção parcial da secreção na luz do brônquio, sendo observada a presença de grande quantidade desta em toda a região distal da árvore brônquica esquerda, com características de molde brônquico. Foi realizada broncoscopia rígida 24 horas após esse procedimento, com adicional remoção do molde brônquico. O material removido durante o procedimento para estudo histológico assumiu a forma cilíndrica ao ser fixado em formalina a $10 \%$, com $4 \mathrm{~cm}$ de comprimento e $0,2 \mathrm{~cm}$ de espessura média. O exame histopatológico revelou grande número de eosinófilos em meio à fibrina (Figura 2). O lavado brônquico realizado durante a endoscopia com broncoscópio flexível foi negativo para pesquisa de BAAR, fungos e exame bacteriológico.

Após os procedimentos endoscópicos, o paciente evoluiu satisfatoriamente, sendo suspensa a ventilação artificial em 72 horas, com alta da unidade de terapia intensiva. $\mathrm{O}$ teste do suor para pesquisa de fibrose cística mostrou-se negativo. A alta hospitalar aconteceu no $10^{\circ}$ dia de internação, com o paciente afebril, eupnéico e sem outras queixas respiratórias.

Foram realizados alguns exames ao final do período de internação: teste do suor para pesquisa de fibrose cística (negativo), prova de falcização (normal), radiografia simples do tórax (normal), avaliação cardiológica (nor-

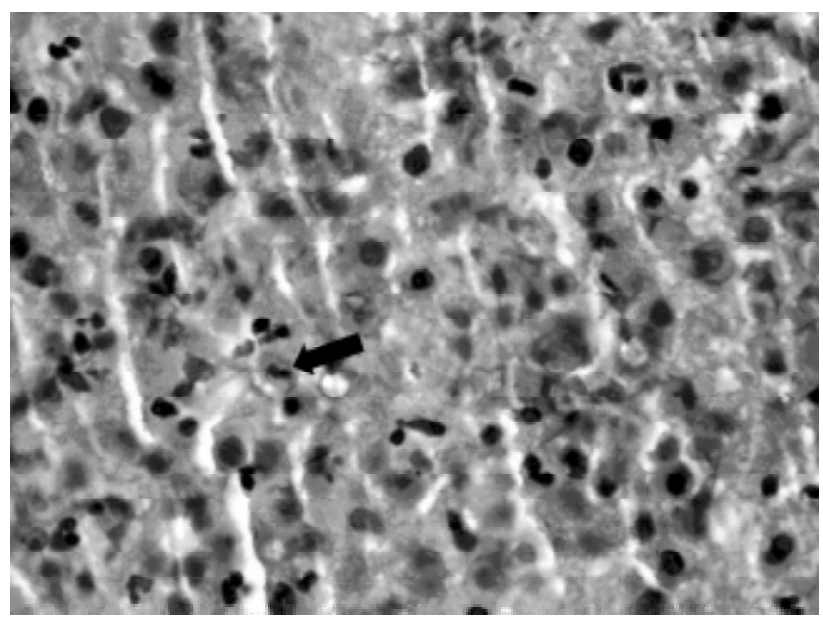

Figura 2 - Exame histológico do molde brônquico evidenciando grande número de eosinófilos em meio à fibrina, debris celulares e outros polimorfonucleares (hematoxilina e eosina, 500X). A seta identifica eosinófilos no corte histológico 
mal). Para complementar a investigação da anemia, foi realizada a eletroforese de hemoglobina, utilizando a técnica de hemolisado com saponina 4 . O resultado mostrou a presença de hemoglobina $\mathrm{H}(\mathrm{HbH})$ em $2 \%$, caracterizando talassemia alfa.

No período de janeiro de 2005 até o momento, o paciente não apresentou recorrência do quadro.

\section{Discussão}

BP é uma condição infreqüente, tendo recebido outras denominações anteriores, tais como bronquite fibrinosa ou croup bronchitis ${ }^{5}$. Geralmente ocorre em doentes com asma, fibrose cística, na anemia falciforme (como síndrome torácica aguda), e no pós-operatório de cirurgia cardíaca ${ }^{6}$. Não havia descrição anterior de ocorrência de BP em portadores de talassemia alfa. É possível que isso ocorra em outros casos, pois, em cerca de $20 \%$ dos casos, a etiologia não está relacionada com as patologias previamente cita$\mathrm{das}^{6}$. A fisiopatogenia da BP em pacientes com anemia falciforme parece estar relacionada com oclusões microvasculares das hemácias falcêmicas, aumento da adesão celular ao endotélio vascular e liberação de citocinas inflamatórias $^{2}$. A talassemia alfa, diagnosticada no presente caso, pode apresentar desde um quadro assintomático até formas variadas de anemia, esplenomegalia e tem suscetibilidade aumentada às infecções.

Uma infecção viral, não pesquisada, pode ter ocorrido nesse paciente. A criança apresentou febre, havia infiltrado intersticial à radiografia de tórax e o cilindro endobrônquico era do tipo I - inflamatório. Um estudo histopatológico realizado por Seear et al. classificou os cilindros brônquicos em dois tipos: tipo I e II ${ }^{7}$. Os cilindros tipo I apresentam predomínio de fibrina, infiltrado eosinofílico e estão mais associados a doenças inflamatórias. Os cilindros tipo II são cilindros com poucas células e compostos por mucina. Estes estão mais associados a pacientes com doença cardiológica. Têm sido propostas, como etiologia para os cilindros do tipo II, causas como baixo débito cardíaco (gerando pressão pulmonar venosa elevada), anormalidades na drenagem linfática e hipersecreção de muco na via aérea ${ }^{8}$.

A BP apresenta manifestação clínica variável e pouco específica, podendo cursar com dispnéia, dificuldade respiratória, tosse, sibilância e febre. A semelhança na apresentação clínica com aspiração de corpo estranho ou asma aguda grave foi descrita previamente por Noizet et al. ${ }^{9}$. Como descrito no presente caso, os sintomas de início agudo, a atelectasia extensa e a ausência de morbidade prévia resultaram em uma impressão inicial de aspiração de corpo estranho. Tal apresentação resultou na imediata indicação de exploração da via aérea por endoscopia. A utilização da broncoscopia flexível foi justificada pela gravidade inicial do quadro e facilidade de realização desse procedimento dentro da unidade de terapia intensiva. O diagnóstico de BP pode ser realizado tanto por broncoscopia quanto pela expectoração de cilindro ou moldes brônquicos 6 .
A terapêutica baseia-se na remoção do molde endobrônquico por endoscopia, muitas vezes suficiente para uma adequada resolução, como no caso em foco. Porém, há propostas de tratamento adicional que podem incluir os corticóides ( $2 \mathrm{mg} / \mathrm{kg} / \mathrm{dia}$, em casos de cilindros tipo I $)^{9}$, antibióticos ${ }^{9}$ para intercorrências bacterianas associadas, lavado broncoalveolar, fisioterapia respiratória e Nacetilcisteína tópica ( 2 a $4 \mathrm{ml}$ da solução a $10 \%)^{10-12}$. Mais recentemente, tem-se proposto o uso de trombolíticos na forma de aerossóis (uroquinase) e instilação endotraqueal de imunoglobulina recombinante (dornase alfa $)^{12}$. A remoção mecânica dos moldes, sob visualização direta (broncoscopia), em crianças com quadros de insuficiência respiratória aguda pode ser bastante útil. A melhor alternativa terapêutica ainda não foi estabelecida em razão da ausência de estudos controlados em uma doença com baixa prevalência.

Casos com apresentação clínica e radiológica semelhante devem alertar o pediatra para a possibilidade do diagnóstico de anemia falciforme ou outras hemoglobinopatias. Devido ao caráter atípico na apresentação, muitas vezes sem anemia severa ou outros sintomas, casos de hemoglobinopatia $\mathrm{H}$ associados com BP podem ter passado desapercebidos. Parece recomendável, com base neste relato, dar uma atenção especial a pacientes com infecção pulmonar e atelectasia no diagnóstico de outras hemoglobinopatias.

A BP é um achado infreqüente em pediatria, mas tem sua importância reconhecida na medida que pode mimetizar diagnósticos como aspiração de corpo estranho e asma aguda grave, estes sim, muito comuns em salas de emergências e unidades de terapia intensiva. Além disso, a busca de doenças subjacentes deve fazer parte da avaliação desse tipo de paciente. A suspeita de BP pelo pediatra, com indicação precoce de broncoscopia flexível em casos como o deste relato, podem tornar o diagnóstico etiológico mais precoce e melhorar o prognóstico dos pacientes com essa condição.

\section{Referências}

1. Bowen A, Oudjhane K, Odagiri K, Liston SL, Cumming WA, Oh KS. Plastic bronchitis: large, branching, mucoid bronchial casts in children. Am J Roentgenol. 1985;144:371-5.

2. Raghuram N, Pettigano R, Gal AA, Harsch A, Adamkievwicz TV. Plastic bronchitis: an unusual complication associated with sickle cell disease and the acute chest pain. Pediatrics. 1997;100: 139-42.

3. Moser C, Nussbaum E, Cooper DM. Plastic bronchitis and the role of bronchoscopy in the acute chest syndrome. Chest. $2001 ; 120$ : 608-13.

4. Naoun PC. Eletroforeses, técnica e diagnóstico. $1^{a}$ ed. São Paulo: Livraria e Editora Santos; 1974.

5. Bowen A. Plastic or cast bronchitis? Am J Dis Child. 1990;144: 1075-6.

6. Brogan TV, Finn LS, Pyskaty DJ Jr, Redding GJ, Ricker D, Inglis $A$, et al. Plastic bonchitis in children: a case series and review of the medical literature. Pediatrics Pulmonol. 2002;34:482-7.

7. Seear M, Hui H, Magee F, Bohn D, Cutz E. Bronchial casts in children: a proposed classification based on the nine cases and a review of the literature. Am J Resp Crit Care Med. 1997;155: 364-70.

8. Quasney MW, Orman K, Thompson J, Ring JC, Salim M, Schoumacher RA, et al. Plastic bronchitis occurring later after the Fontan procedure: treatment with aerosolized urokinase. Crit Care Med. $2000 ; 28: 2107-11$. 
9. Noizet O, Leclerc F, Leteurte S, Brichet A, Pouessel G, Dorkenoo A, Fourier C, Cremer R. Plastic bronchitis mimicking foreign body aspiration that needs a specific diagnostic procedure. Intensive Care Med. 2003;29:329-31.

10. Costello JM, Steinhorn D, McColley S, Gerber ME, Kumar SP. Treatment of plastic bronchitis in a Fontan patient with tissue plasminogen activator: a case report and review of the literature. Pediatrics. 2002;109:e67.

11. Durward A, Forte $V$, Shemie SD. Resolution of mucous plugging and atelectasis after intratracheal rhDNase therapy in a mechanically ventilated child with refractory status asthmaticus. Crit Care Med. 2000;28:560-2.
12. Manna SS, Shaw J, Tibby SM, Durward A. Treatment of plastic bronchitis in acute chest syndrome of sickle cell disease. Arch Dis Child. 2003;88:626-7.

Correspondência:

Paulo Márcio Condessa Pitrez

Av. Ipiranga, 6690

Instituto de Pesquisas Biomédicas da PUCRS, $2^{\circ}$ andar

CEP 90610-000 - Porto Alegre, RS

Tel./Fax: (51) 3384.5104

E-mail: pmpitrez@pucrs.br 